\title{
Intervención de terapia ocupacional en prevención y tratamiento de obesidad y diabetes mellitus tipo II: "nunca pensamos que era algo más que una dieta"[1]
} Porras A., Ximena ${ }^{[1]}$; Cáceres N., Miluska ${ }^{[2]}$; Garcés M., Alejandra ${ }^{[3]}$; Riveros G., Maria Francisca ${ }^{[4]}$;
Seguel V., Alejandra ${ }^{[5]}$

\section{- Resumen}

El presente artículo muestra la experiencia de intervención de Terapia Ocupacional con personas Obesas y Pre Diabéticas, realizado en un Centro de Salud Familiar de la Comuna El Bosque.

La experiencia se enmarca dentro de un Programa de Intervención Integral, para personas con Obesidad y Pre Diabetes, financiado por el Fondo Nacional de la Salud FONASA y desarrollado como una experiencia piloto en cinco establecimientos de salud durante el año 2004 Y 2005, experiencia que por sus resultados en el presente año, está siendo implementada a lo largo de todo Chile.

La intervención, que considera el abordaje integral de las patologías antes señaladas, es llevada a cabo por un equipo multiprofesional compuesto por: Nutricionista, Médico, Terapeuta Ocupacional, Kinesiólogo y Auxiliar Paramédico.

La experiencia en la práctica fue desarrollada por una Terapeuta Ocupacional, miembro del equipo del Centro de Salud Familiar y docente de la Universidad Andrés Bello, y cuatro alumnas en práctica de la Carrera de Terapia Ocupacional, de la misma casa de estudios, quienes realizaron intervenciones individuales y grupales por espacio de 10 meses, entre Julio de 2004 y Abril de 2005.

Lo que se presenta en este artículo, así como el análisis y reflexiones que de éste surgen, constituyen un espacio innovador y pionero para la Terapia Ocupacional como disciplina y abren un camino para la inserción del Terapeuta Ocupacional en la prevención y tratamiento de patologías crónicas, así como la promoción de la salud en la atención primaria.

Palabras Clave: Terapia Ocupacional, Obesidad, Diabetes Mellitus, Nutricion, Hábitos.

\begin{abstract}
- Abstract
The present article is about the experience of working in Occupational Therapy with fat and pre-diabetic people, carried out in a Family Health Center in El Bosque commune. This knowledge is applied to a program of integral intervention to pre-diabetic and fat people, subsidized by FONASA National Funds of Health and realized as a pilot experience in five different establishment of health between 2004 and 2005. For its excellent results this experience in the current year is being applied to others communes of our country.
\end{abstract}


The intervention that considers the complete attention of the pathologies before mentioned is performances by a multiprofessional team integrated by Nutritionist, Doctors, Occupational Therapist, Kinesyologist and Paramedical Assistant.

Interventions that, in the practice were developed by an Occupational Therapists member of the team of the Family Health Center and teacher from Andres Bello University, and four students making practicing in the occupational therapy career from the some establishment, who carried out individual and group interventions approximately ten months between July 2004 and April 2005.

The analysis and reflections we can observe in this paper constitute an innovative and pioneer space for the occupational therapy as a discipline, in the prevention and treatment of the chronic pathologies, and to the health promotion in the primary attention.

Key Words: Occupational Therapy, Obesity, Diabetes, Nutrition, Hábits.

\footnotetext{
${ }^{[1]}$ Terapeuta Ocupacional, Magíster en Docencia Universitaria, Miembro del Equipo del Centro de Salud Familiar Canciller Orlando Letelier, Instructora de Practica Profesional y Profesor Adjunto de Pre y Post Grado de la Carrera de Terapia Ocupacional de la Facultad de Ciencias de la Rehabilitación de la Universidad Andrés Bello $[2,3,4,5]$ Alumna de $4^{\circ}$ año de la Carrera de Terapia Ocupacional de la Universidad Andrés Bello

Contacto > > ${ }^{[1]} \mathrm{E}-\mathrm{mail}$ : toporras@tutopia.com; Fono: 09- 8635482; ${ }^{[2]} \mathrm{E}$-mail: miluandrea84@hotmail.com; Fono:08-3702202; ${ }^{[3]}$ al.garces@uandresbello.edu; E-mail: 09-8375882; ${ }^{[4]}$ E-mail: fran.riveros.g@hotmail.com; Fono: 08-9491748; $>{ }^{[5]}$ E-mail: al.seguel@gmail.com; Fono: 08-9156111
} 


\section{- Antecedentes epidemiológicos}

La nutrición humana constituye uno de los determinantes más importantes de la salud y bienestar de las personas. El adecuado equilibrio entre la calidad y la cantidad es esencial para el crecimiento y desarrollo ${ }^{(1)}$. Sin embargo, podemos observar cómo han aumentado considerablemente las enfermedades y síntomas relacionados con la malnutrición por exceso en nuestra población, razón por la cual se hace indispensable intervenir con un enfoque preventivo y promocional en éstas áreas.

En nuestro país, una serie de factores han llevado a nuestra población a cambiar sus hábitos alimenticios. El mejoramiento económico ha significado cambiar el estilo de alimentación hacia una dieta caracterizada por alto consumo de alimentos procesados, con comida rápida rica en grasas saturadas y altamente calóricos. El consumo de grasas ha aumentado de $13.9 \mathrm{Kg}$./persona/año en 1975 a $16.7 \mathrm{Kg} . /$ persona/año en 1995. El consumo de azúcar también se incrementó de 30.2 a $39.2 \mathrm{Kg} . /$ persona/año en el mismo periodo.

Los sistemas de información del estado nutricional de la Junta Nacional de Auxilio Escolar y Becas (JUNAEB), que reflejan el estado nutricional de los niños y niñas usuarias del sistema, demuestran un incremento importante de la obesidad en niños en los últimos años.

En otros grupos de edad, la situación no es muy diferente. El estudio Interhealth Chile ${ }^{(2)}$ muestra cómo en mujeres, la prevalencia de sobrepeso ha aumentado considerablemente, junto con la de obesidad. La media del índice de Masa Corporal de las mujeres ha ido subiendo paulatinamente; así en 1988 era de 25,5, en 1992 de 27,0 y en 1997 de 28,4.

Por otra parte, la Diabetes Mellitus tipo 2 (DM2) abarca al $90-95 \%$ de todas las personas con diagnostico de DM. Este porcentaje en Chile, de acuerdo al estudio Diabetes y Calidad de la Atención, es de $94,4 \%$. Este tipo de DM se asocia tanto a factores de riesgo modificables: obesidad e inactividad física, como a factores no modificables: factores genéticos e historia familiar positiva, envejecimiento, diabetes gestacional, tolerancia a la glucosa alterada y raza/etnia ${ }^{(\underline{1})}$.

La tasa de mortalidad ajustada por edad por DM en Chile, es de 14 por 100.000 habitantes para 1999, ocupando el $8^{\circ}$ lugar de todas las causas de muerte. Entre 1985 y 1999 la tendencia de esta tasa, ajustada por edad, es ascendente con un aumento de un $25 \%$, que no se explicaría por el envejecimiento de la población. Las proyecciones realizadas con un modelo exponencial muestran que esta tasa seguirá aumentando los próximos 10 años. ${ }^{(\underline{1})}$

EI MINSAL ha propuesto una serie de medidas respecto al tema de la obesidad y los factores de riesgo cardiovascular, que pretenden hacer frente a este aumento, entre ellas podemos mencionar: incorporación de programas de tratamiento de la obesidad en la Atención Primaria de Salud (APS), implementación del Programa Alimentación Sana y Vida Activa en escolares y embarazadas, regulación en el control de alimentos y campañas comunicacionales que promuevan una alimentación saludable.

El aumento de las patologías por malnutrición y el mínimo impacto de las medidas sanitarias realizadas al respecto nos hace pensar en la necesidad urgente de abordar el problema del sobrepeso y la obesidad desde un enfoque más integral, ya que ella no 
depende sólo de factores genéticos y relacionados con el metabolismo, sino que los factores ambientales son determinantes en los hábitos y conductas alimentarias de un individuo o familia en particular.

Ahora, estos factores ambientales no incluyen sólo la conducta alimenticia y la forma en que esta es llevada a la práctica. Es fundamental, en este sentido, hacer hincapié en aquellos factores culturales, sociales, políticos y económicos (por mencionar algunos), que llevan a un individuo, a una familia, y en definitiva, a una comunidad a alimentarse de determinada manera.

\section{- Introducción al proyecto "}

El año 2004 el Ministerio de Salud y FONASA se dieron a la tarea de desarrollar un modelo piloto de intervención en obesidad que permitiera establecer un programa que a futuro se implementase de manera estable en la APS. Así entonces, la propuesta consistió en desarrollar un programa de intervención con enfoque preventivo y promocional, centrado en el desarrollo de estilos de vida saludables en adultos obesos y prediabéticos. El propósito era modificar los estilos de vida de los usuarios intervenidos, a través de evaluaciones sucesivas, seguimiento nutricional, apoyo emocional, educación y asesoramiento, con la finalidad de reducir el riesgo de DM II y mejorar su calidad de vida.

Es en este contexto, y dada la experiencia previa del equipo profesional del Centro de Salud en Obesidad y enfermedades asociadas a riesgo cardiovascular, que se desarrolla la propuesta de realizar una intervención centrada en los hábitos y su sustento emocional, social y cultural.

La propuesta de atención consiste en: cuatro meses de intervención, exámenes de laboratorio al inicio y al $4^{\circ}$ mes, tres consultas por médico, cuatro consultas por nutricionista, 14 sesiones de actividad física y cuatro sesiones grupales de carácter educativo-terapéutico realizadas por Terapeuta Ocupacional.

Las características que deben cumplir los usuarios que ingresen a este proyecto son las siguientes:

Adultos entre 18 y 45 años, en los que se pesquisen factores de riesgo asociados a la DM, a través del Examen de Salud Preventivo del Adulto (ESPA). Estos Factores de Riesgo son:

- Encontrarse en sobrepeso u obesidad, con IMC entre 25 y 35.

- Con glicemia en ayunas entre 100 y 125 mg/dl.

- Sin otros factores de riesgo cardiovascular.

Los 70 participantes del proyecto aquí presentado, fueron derivados desde los consultorios de atención primaria de toda la comuna de El Bosque mediante una interconsulta realizada por médico o Nutricionista.

Cabe señalar, que el equipo de salud antes señalado, compuesto por: Nutricionista, Terapeuta Ocupacional, Kinesiólogo, Médico y Auxiliar Paramédico, ya tenía una 
historia de más de 3 años trabajando experiencias locales en el tema de Obesidad, Hipertensión Arterial y Diabetes Mellitus, con un enfoque integral, familiar y comunitario. Por eso, la propuesta de ser un establecimiento Piloto en el Proyecto de FONASA, se transformó en una oportunidad para validar ese enfoque y generar propuestas más globales.

\section{"Intervención de terapia ocupacional "}

Para comenzar es importante señalar que la intervención desarrollada, tanto en el ámbito individual como grupal, toma elementos desde al menos cuatro Marcos o Enfoques Teóricos, estos son:

- Modelo de Salud Familiar

- Enfoque Familiar Sistémico(ㄱ)

- Modelo Ecológico(4)

- Modelo de Ocupación Humana( ${ }^{(\underline{5})}$

Como Marcos Metodológicos para la Intervención se consideran:

- Modelo de Educación Popular(모

- Teoría de la Comunicación Humana ${ }^{(\underline{7})}$

La propuesta de intervención desde la Terapia Ocupacional se sustenta, por una parte, en la idea de comprender tanto a las personas usuarias y participantes del proyecto, como al equipo de salud que interviene, como miembros de una arquitectura compleja de sistemas como son: la familia, la comunidad, organizaciones e instituciones presentes en su medio social, el sistema laboral, el sistema de salud, los medios de comunicación, el sistema político y económico, la cultura y el medio ambiente físico, entre otros. En ellos, todos somos "todo y partes" al mismo tiempo, así entonces, podemos visualizarnos como "integrantes, integrados e interactuantes" con múltiples sistemas $^{(\underline{8})}$. Estos sistemas, impactan e influencian a las personas y determinan 0 condicionan en gran medida las conductas y las relaciones que se establecen al interior de esta compleja "arquitectura de sistemas", en la que muchas veces, los mensajes, significados y representaciones de un mismo fenómeno o conducta pueden ser diametralmente opuestos, dependiendo de cual sea el "foco desde donde éste se esté mirando", lo que conflictúa y confunde a las personas que participamos en ellos. Sin olvidar las relaciones e interacciones que surgen entre éstos, y que conforman lo que Morín( ${ }^{(\underline{)})}$ denomina "el ecosistema", y que se constituyen en nuevos "sistemas de sistemas", que implican nuevas relaciones y nuevas interpretaciones mucho más complejas.

Para el individuo el principal sistema lo constituye su familia siendo el espacio donde se desarrollan los aspectos cognitivos y afectivos, donde también se establecen las relaciones, en un "campo emocional", a través de la comunicación verbal y no verbal, donde las conductas que llevan a cabo las personas constituyen "formas de comunicarse", que tienen un significado para quien las practica, para su familia y para los sistemas con los que se relaciona.

Al respecto, la Teoría de la Comunicación Humana ${ }^{(\underline{7})}$ señala que es imposible no comunicar; toda conducta en una situación de interacción tiene un valor de mensaje, 
es decir, es comunicación, se deduce que, por mucho que uno lo intente, no puede dejar de comunicar; actividad o inactividad, palabras o silencios tienen siempre valor de mensaje e influyen sobre los demás, quienes, a su vez, no pueden dejar de responder a tales comunicaciones y por ende también comunican.

Por otra parte, todas las personas traen consigo una herencia biológica. Ampliamente estudiadas están también las predisposiciones genéticas a muchas enfermedades tales como la obesidad, la diabetes, la hipertensión arterial, las enfermedades reumáticas, el alcoholismo y el cáncer, entre otras. Sin embargo conjuntamente con estas predisposiciones están las herencias o transmisiones en el campo emocional, lo que Bowen denomina "transmisión multigeneraciónal". Es así entonces, que muchas de nuestras relaciones estarían influenciadas por formas de relacionarse que se dieron en nuestra familia de origen o antes, y que continúan operando en el campo emocional de nuestras relaciones actuales ${ }^{(\underline{3})}$, lo que es extrapolable también a las conductas y los hábitos relacionados con la alimentación.

Es fundamental también, considerar el contexto socioeconómico y cultural donde se desenvuelve la familia. Las frustraciones de las clases populares penetran el subsistema familiar, llevándolo en muchos casos a la entropía ${ }^{(10)}$. Si visualizamos la conducta alimenticia, y la "comida" como una importante fuente de satisfacción y, a su vez, un elemento por donde se canaliza mucha de la ansiedad y la angustia sentida por las personas y las familias en los sectores populares, podemos ver cómo ésta debe ser interpretada y analizada mucho más allá de la mera "conducta" y necesariamente, debemos orientar nuestro análisis e intervención a todos aquellos aspectos antes mencionados, que son los que la sustentan, la potencian, la mantienen y la retroalimentan.

En nuestro trabajo con familias de la comuna El Bosque, hemos escuchado innumerables veces frases tales como: "yo pasé mucha hambre en mi infancia, ... y no voy a permitir que mis hijos pasen por lo mismo". Podemos ver entonces, como una serie de hábitos evaluados por el equipo de salud como "no saludables" o factores/conductas de riesgo, como son: comprar golosinas a los niños, alimentarlos excesivamente y por consiguiente crear hábitos alimenticios poco saludables, se fundamentan, -por lo menos en un nivel afectivo/emocional y en lo que se refiere al significado y representación que le otorgan quienes llevan a la práctica estas conductas $^{(\underline{1})}$-, en compensar una serie de carencias y frustraciones de la historia de vida, a través de la alimentación, sin olvidar que este mismo mecanismo lo podemos extrapolar a otra serie de conductas y hábitos en otros aspectos de la vida cotidiana.

Es sabido por el equipo de salud que para lograr un cambio en la conducta alimentaria de nuestros usuarios, requerimos promover un cambio de hábitos, para que este cambio ocurra se requiere de la toma de conciencia del individuo acerca de su situación de salud-enfermedad y que de esta manera logre comprender cual es su responsabilidad en la mantención de su calidad de vida.

El fin de cada individuo es sentirse bien, funcionar y participar del mundo que lo rodea con el menor numero de problemas posibles. Sin embargo: ¿por qué entonces tenemos hábitos de vida no saludables?

La Terapia Ocupacional, presume a la ocupación como parte de la condición humana. Allí distinguimos las áreas del quehacer como: Actividades de la Vida Diaria (Básicas e Instrumentales), actividades de trabajo, educación, juego, ocio y participación 
social ${ }^{(12)}$. Y organizamos nuestras ocupaciones a través de roles, rutinas y hábitos de comportamiento. Todo ello partiendo de la base, de que esta organización -particular y única de cada ser humano- se sustenta en base al sentido y la significancia personal que cada uno le otorgue. En ese sentido, cada uno cuenta con habilidades, destrezas, motivaciones e intereses personales, que utilizamos en nuestra relación con el medio ambiente que nos rodea, relacionándonos con él a través de la ocupación.

Definimos hábitos como: aquellas tendencias latentes, adquiridas de previas repeticiones, que operan principalmente a nivel subconsciente e influencian nuestros patrones de comportamiento( ${ }^{(12)}$. Los Hábitos, se pueden entender como un estilo de comportamiento, o un típico estar en el mundo ${ }^{(5)}$. Ellos organizan nuestras acciones y comportamientos, dándole un sentido particular y único a nuestro actuar en el mundo.

Por lo dicho anteriormente, cambiar un hábito es tarea difícil, está arraigado en nuestro accionar y opera casi en forma automática.

Sin embargo, en la medida que tenga un sentido para la persona y éste sea visto en un contexto ocupacional real, vinculado a las necesidades de la persona, es posible cambiarlo.

Los hábitos no se cambian a través de un consejo, ni en una relación de nivel teórica o académica. Los hábitos son parte de nuestra forma de relacionarnos con el ambiente y, por lo tanto, sólo se pueden modificar desde una perspectiva basada en la Ocupación. Nuestra propuesta de intervención apunta a ello.

Como ya mencionamos en extenso, existen una serie de factores ambientales, sociales y culturales que determinan lo que las personas entienden acerca de la salud y la enfermedad, factores, que tienen que ver con nuestra historia familiar y nuestras experiencias, y que determinan emociones tales como el miedo, la angustia, la confianza o la ansiedad frente a ciertos temas. De esta manera, la "interpretación" que cada uno hace de lo que ve y escucha está relacionado con esas emociones.

Por eso, es fundamental tener en cuenta estos factores a la hora de tratar de compartir con nuestros usuarios aquellos "temas de salud" que para nosotros son tan importantes y tan familiares. Al referirnos a este proceso como "compartir", y no enseñar ó educar, estamos partiendo desde la premisa básica acerca de que el cambio es un proceso personal y único en cada ser humano, y que para que sea real, éste debe partir de la propia voluntad y sustentarse en un significado personal y no heterodirigido.

Demás está decir que cada uno de los que participa en esa relación -el médico o profesional de salud y el paciente/usuario- tienen claras intenciones de obtener un beneficio de ese proceso, es decir, la persona viene solicitando ayuda y su necesidad es "mejorarse o sentirse mejor", y el médico o profesional quiere ayudarlo, "sanarlo" o mejorar su calidad de vida. Sin embargo entonces, ¿porqué es tan difícil que los usuarios lleven a la práctica lo que les dice el equipo de salud?, o, ¿por qué las personas no cambian sus hábitos de vida no saludables de manera espontánea?

Nuestro foco de intervención tiene relación con la conducta alimentaria, los hábitos alimenticios y el estilo de vida de las personas, lo que impactaría en su calidad de vida y condición de salud-enfermedad. Es por ello que la intervención antes señalada 
apunta a "cambiar o modificar" los hábitos alimenticios y el estilo de vida, a fin de que éste no desencadene en conductas nocivas o dañinas para la salud de las personas.

Pensemos en lo que ocurre con el hábito alimentario de comer pan en el almuerzo; la persona que lo lleva a cabo, esta interactuando con múltiples sistemas, para los cuales esta misma conducta representa significados y resultados diferentes, por ejemplo: un hombre de 45 años, obeso, que se come dos "marraquetas" en el almuerzo. Mientras para el equipo de salud, esta conducta es nociva, riesgosa e inadecuada, para el usuario y su familia esta conducta es validada colectivamente, tiene un significado a nivel personal, social y cultural (entre otros), y fue adquirida en un proceso paulatino y progresivo, por lo que en la actualidad no representa un acto "reflexivo y consciente", sino más bien una conducta automática e irreflexiva que se lleva a cabo diariamente. En su familia, esta conducta representa un hábito, tanto para él como para los otros miembros de su grupo familiar, que, aunque no la practiquen, la reconocen en él como un habito, por lo que además le otorga un sentido de identidad particular dentro de este grupo -"mi papá no puede almorzar sin pan"-. Desde el punto de vista cultural, también representa una conducta validada y aceptada socioculturalmente, dado que en nuestro país el consumo de pan en las diferentes comidas diarias es algo habitual. Económicamente también es algo importante para su familia, y principalmente para la dueña de casa, ya que cuando no hay pan, la familia tiende a ingerir mayor cantidad de alimentos y de esa manera la comida no alcanza para todos. Es decir, sólo en este nivel de análisis podemos ver cómo la misma conducta tiene un significado diametralmente opuesto para un equipo de salud y para una familia.

Acá el punto no es llegar a dilucidar quién tiene la razón, el foco del análisis debe dirigirse a la relación, tanto entre el individuo, y sus hábitos, como entre el usuario y el equipo de salud que propone el cambio de hábitos. La invitación es a preguntarnos: ¿para qué?, ¿para qué queremos promover un cambio en los hábitos de nuestros usuarios?, así como también, -si ese cambio fuera necesario y significativo para la vida de un usuario- ¿quién es el responsable del cambio?, ¿quién lo inicia? y ¿cómo lo lleva a la práctica?.

Nuestra intervención se focaliza principalmente en aquellos aspectos que históricamente han sido dejados de lado por las intervenciones tradicionales en lo que a estilos de vida y hábitos alimenticios se refiere.

Es en estos aspectos donde debemos poner el énfasis cuando trabajamos en Educación y Promoción de la Salud si queremos que nuestro objetivo de mejorar la calidad de vida de nuestros usuarios se cumpla.

Entonces, para que el proceso de educar en salud tenga impacto en nuestros usuarios debemos buscar estrategias y metodologías que tomen en cuenta esos aspectos y de esa manera nos aseguren un buen resultado.

Nuestra propuesta entonces consiste en una metodología participativa basada en la Educación Popular y con un enfoque Comunicacional y Sistémico, donde el conocimiento es el RESULTADO de un PROCESO, y donde las EXPERIENCIAS y EMOCIONES de los participantes representan tanto el punto de partida como el punto de llegada, y se convierten en nuestra principal preocupación y foco de intervención. 


\section{Metodologías de intervención}

La intervención de Terapia Ocupacional consiste en:

- Una Entrevista de Evaluación y Motivación Individual

- Cuatro Sesiones de Taller Grupal

La entrevista individual tiene por objetivo generar un vínculo terapéutico de confianza y entrega mutua entre el Terapeuta Ocupacional y el usuario, como también obtener información, a modo de insumo, para las sesiones grupales.

En relación a las sesiones educativas grupales, irán enfocadas a promover un cambio de hábitos en los usuarios. Para que esto ocurra se requiere de la toma de conciencia del individuo acerca de su situación de salud - enfermedad y que de esta manera logre comprender cual es su responsabilidad en la mantención de su calidad de vida.

La modalidad de trabajo será de tipo participativa y experiencial, en donde los conocimientos se van "construyendo" en conjunto con los participantes ${ }^{(\underline{13})}$. Este taller debe ser voluntario a fin de favorecer la autonomía y responsabilidad personal en el autocuidado y evitar la dependencia con los profesionales o el sistema de salud.

El grupo de trabajo de Terapia Ocupacional está conformado por una Terapeuta Ocupacional y 4 Co-Terapeutas (estudiantes que cursan el $4^{\circ}$ semestre de la carrera en la Universidad Andrés Bello).

El horario en que se realizaron las sesiones tanto individuales como grupales fue los días Lunes o Miércoles entre las 18.00 hrs. hasta las 20.00 hrs. aproximadamente; horario que facilita la permanencia y adherencia de los usuarios al proyecto. También se realizaron algunas actividades los días sábado en la mañana.

\section{Objetivos generales}

- Facilitar en los participantes un proceso de toma de conciencia y autonomía frente a su situación de salud en relación a la significancia personal de sus hábitos alimentarios.

- Que los beneficiarios de este programa logren reconocer y descubrir el sentido personal de sus hábitos alimentarios en un contexto histórico, familiar y transgeneracional.

\section{- Objetivos específicos "}

- Que los participantes adquieran herramientas para el desarrollo de nuevos hábitos, en un contexto individual y familiar.

- Promover un proceso de reflexión, evaluación y autocrítica en los usuarios que favorezca un cambio hacia un estilo de vida saludable.

- Que los participantes identifiquen y asocien factores ambientales, emocionales y relacionales a su conducta alimentaria. 
- Fundamentos de la entrevista individual .

La importancia de la entrevista individual radica en lograr un vínculo entre el Terapeuta y el usuario, evaluar el contexto individual, familiar y comunitario de éste, informarle acerca del proceso que implica su participación en el programa, y obtener insumos para las sesiones grupales tales como: datos relevantes del contexto familiar, sintomatología más frecuente, hábitos de vida y rutinas, entre otros.

Otro de los objetivos de la entrevista individual es favorecer el proceso de toma de conciencia y auto responsabilidad por parte de los participantes, con el fin de favorecer y potenciar la motivación y el compromiso con las actividades del programa y establecer una relación de mutua confianza y colaboración entre profesional y usuario.

El contexto físico juega un rol importante para fomentar la confianza. El inmobiliario se dispone de tal forma que se genere un ambiente acogedor; el escritorio no separa al Terapeuta del usuario y es él quien decide en qué lugar tomar asiento. Esto favorece de alguna manera, que el usuario no se sitúe en una posición o rol de "paciente", sino por el contrario, favorece una relación más horizontal y un rol participativo, aspectos fundamentales a lo largo de la entrevista.

Desde un comienzo, la relación que se establece entre T.O. y usuario no es jerárquica. Se utiliza un lenguaje simple (sin términos clínicos pero sin caer en lo coloquial) con el fin de que la persona comprenda lo que se le explica, favoreciendo el desarrollo de una conversación fluida y en confianza. Por otra parte, el delantal no forma parte de la vestimenta del T.O. con el objetivo de eliminar la barrera que distingue al profesional del paciente.

El tiempo de duración de la entrevista corresponde a 45 minutos, el que puede variar dependiendo de las necesidades y características de cada usuario.

\section{- Ítems de la entrevista individual "}

\section{Evaluación a través de Genograma}

El Genograma es una representación gráfica del contexto familiar del entrevistado(a), a través de él se pueden evaluar y representar de manera gráfica las complejas dinámicas familiares, normas y "formas" en que se relacionan los miembros de una familia. Aporta a la intervención una visión global, rápida y sistémica de la realidad del usuario y su entorno familiar, tanto en su familia nuclear, como en la familia de origen, donde se incluyen al menos tres generaciones a fin de obtener información importante y extensa que aporte en análisis posterior ${ }^{(14)}$.

El Genograma, en este caso resulta ser una rica fuente de hipótesis sobre cómo la obesidad, el sobrepeso y otra serie de factores de riesgo asociados pueden estar relacionados con el contexto y la dinámica familiar, permitiéndonos también observar la evolución y curso que estos factores han tenido en el contexto familiar a través del tiempo. 
Este instrumento de evaluación además nos permite, entre otros, obtener información acerca de la situación socioeconómica, educacional y habitacional del individuo. Y por las características del programa y las áreas a intervenir, además es utilizado por el profesional para reconocer el curso y la evolución histórica y genética que han seguido algunas patologías y factores de riesgo presentes en el grupo familiar tales como: diabetes mellitus e hipertensión arterial, factores de riesgo cardiovascular y tabaquismo entre otros, así como otros factores sociales, culturales y económicos que repercuten en la salud del individuo.

\section{Evaluación de Rutina}

Llevar a cabo acciones coordinadas, sencillas o complejas para planificar y dirigir las demandas de las obligaciones o tareas diarias, corresponde a la definición de rutina. Esta es de gran valor ya que permite identificar hábitos propios de cada individuo y cómo estos inciden en su salud. La rutina se analiza con el objetivo de detectar cómo opera y de qué manera se lleva a cabo el patrón de hábitos del usuario. Hábitos que pueden ser útiles para el funcionamiento ocupacional diario, o que pueden representar un riesgo para la salud del individuo o para su entorno.

A través de la evaluación de la rutina, se hace hincapié en evaluar la existencia y frecuencia de hábitos y conductas de riesgo para el usuario, entre los cuales se encuentran: hábitos desorganizados, demasiado rígidos, conductas ansiosas, etc. A partir de su rutina se observan roles, patrones dentro del desempeño ocupacional, y motivaciones, factores de gran relevancia para la intervención, determinantes en el resultado del tratamiento.

\section{Evaluación de las áreas del desempeño ocupacional}

El hombre, es un ser activo que influencia su estado de salud física y mental a través de la ocupación. Las ocupaciones permiten cubrir sus necesidades individuales y grupales. Esto quiere decir que la ocupación sirve a las personas y no estás a la ocupación. Por lo tanto, es de vital importancia conocer las actividades recreativas, sociales y productivas de la persona, ya que se relacionan entre si permitiendo un equilibrio en su vida, favoreciendo la adquisición de habilidades, el desarrollo de intereses y la organización de la rutina, dando a la persona un sentido de eficacia significativa en su entorno (realidad).

A su vez, las características particulares de las actividades de educación, trabajo y uso del tiempo libre influyen directamente en los hábitos alimenticios de las personas, es por ello que en este punto de la entrevista, aparece información relevante en relación a cómo las condiciones laborales y otros factores socioeconómicos influyen fuertemente en lo que los usuarios pueden o no hacer respecto de su propia alimentación, determinado, muchas veces, por factores externos y ajenos a su voluntad o motivación. Por ejemplo, podemos citar algunas frases textuales de los usuarios que hacen referencia a lo anterior: "mi horario de trabajo no me permite almorzar" o "mi horario de colación es de sólo unos minutos y como alimentos muy poco nutritivos, etc. 


\section{Evaluación de Redes Sociales}

La Red Social se define como el conjunto de vínculos o conexiones que se establecen entre instituciones y/o personas a lo largo del tiempo, y está orientado hacia el intercambio de apoyo social (afectivo, emocional, financiero, servicios, etc.). Sus relaciones se dan en ámbito familiar, de amistades, laboral y comunitario dentro de un contexto histórico determinado.

Como ya hemos dicho antes, la calidad de vida involucra no sólo al individuo, sino también a la familia, comunidad y la sociedad en la que participa, los cuales componen lo que son sus redes sociales. Estas influyen en la forma en que el usuario resuelve sus problemas y en la evolución de éste a lo largo del tratamiento.

A partir de las redes sociales se puede caracterizar una población, ya sea en cuanto a sus intereses, creencias, objetivos y metas.

Las redes sociales permiten generar relaciones de colaboración, poner en común recursos, desarrollar actividades en beneficio de los individuos, ampliar y estrechar vínculos, crear sentido de pertenencia, socializar conocimientos y experiencias, reconstituir la confianza social y establecer relaciones de intercambio y reciprocidad.

De esta manera, las redes sociales constituyen un factor protector del individuo, ya que frente a alguna dificultad, contará con el apoyo y colaboración de un grupo de personas e instituciones.

En este proyecto es fundamental la participación del individuo, pero también lo es contar con el respaldo de las redes sociales, de esta forma, el usuario se sentirá apoyado y contenido a lo largo de todo el proceso.

\section{Evaluación de Sintomatología Asociada}

Permite identificar los factores que motivan al usuario a ingresar al proyecto, entre los cuales se encuentran: estética, salud, baja autoestima, factores genéticos y conductas molestas para el individuo, relacionadas con sus hábitos alimenticios, y que influyen en el desempeño de su vida diaria. Factores que incidirán en su compromiso y adherencia a lo largo del tratamiento.

Este ítem influencia, a su vez, el proceso de toma de conciencia, por parte del usuario, acerca de los elementos que inciden en su situación de salud actual.

Por otro lado, provee al Terapeuta Ocupacional de insumos necesarios para el desarrollo de las sesiones grupales. Estos, insumos, corresponden al "por qué" el usuario decide ingresar al proyecto y a las vivencias que éste experimenta con la comida y su relación con ésta.

\section{Evaluación de la Historia de Vida}

En la intervención de Terapia Ocupacional, la historia de vida es esencial. Esta entrega datos relevantes sobre el usuario, obteniendo información acerca de vínculos afectivos, relaciones intra familiares, vivencias y experiencias significativas. Datos de gran importancia en el abordaje individual del tratamiento. También otorga información 
sobre la calidad de vida a lo largo de su historia, en cuanto a nivel socioeconómico, acceso a oportunidades, realización personal, vida afectiva y proyectos personales.

La historia personal y familiar, favorece un proceso continuo de reflexión y análisis para la persona, construyendo su propio concepto de salud y calidad de vida, a partir de su experiencia. El relato de la historia personal y familiar, favorece un proceso casi de reflexión para la persona, reconociendo por ejemplo: sucesos que marcaron su vida y que pueden tener cierto grado de responsabilidad en su salud actual.

\section{"Fundamentos de la intervención grupal "}

\section{Aspectos Metodológicos}

Se trabajará con una metodología de trabajo participativa, de tipo "experiencial" en donde los conocimientos se van "construyendo" en conjunto con los participantes a partir de la experiencia personal y las actividades realizadas dentro del taller.

Las actividades a realizar son fundamentalmente lúdicas, como dinámicas, juegos y actividades de reflexión y análisis que favorezcan la integración de los conocimientos y el aprendizaje desde un ámbito primordialmente emocional y afectivo, más que desde lo cognitivo o intelectual.

Tomamos como Marcos de Referencia Metodológicos y Operacionales el Modelo de Ocupación Humana ${ }^{(\underline{5})}$ y la Educación Popular ${ }^{(\underline{6})}$. Se trabaja desde la experiencia personal y colectiva en un proceso en el cual el cambio surge desde el propio individuo, en su relación con el ambiente familiar y social, como una necesidad sentida.

Utilizamos una metodología participativa, basada en la concepción de salud como un proceso continuo, que requiere de la motivación inicial de las participantes por el cambio (o no cambio) en su situación de salud.

Por todo lo anterior la participación en el taller debe ser de carácter voluntario. Así, el equipo de profesionales tratantes (Médico, Nutricionista, Kinesiólogo, Terapeuta Ocupacional y Equipo Técnico Administrativo), les "ofrece" a los usuarios la posibilidad de participar del taller como parte del programa de intervención, explicándole de qué se trata y cuáles serían los beneficios que esto le podría otorgar a ella y su familia.

En cada sesión se intenta que los usuarios relacionen sus experiencias personales y familiares con el tema a tratar, de manera que las estrategias que surjan, estén basadas en sus propias experiencias y las que se desarrollen a partir del trabajo grupal.

Así, los contenidos del taller se entregan no de manera expositiva, ni como una charla, sino que se van generando en forma reflexiva entre las terapeutas y el grupo durante las sesiones a través de una serie de herramientas tales como: dinámicas y juegos, medios visuales, plenarios, entre otras. En ese sentido, es muy importante destacar también que el lenguaje utilizado durante las sesiones es simple y cotidiano, cuidando 
no caer en la utilización de términos médicos o científicos que sean difíciles de entender por los usuarios o que contribuyan a situarlos en una posición de "pacientes".

Todas estas técnicas nos permiten una aproximación a sus problemas desde lo emocional, a partir de las vivencias y experiencias de cada una. De no ser así caeríamos sólo en la "racionalización" de los problemas, lo que favorece la adopción de una serie de "mecanismos de defensa" e impide un "darse cuenta" o "comprender" a través de la practica, así como también enriquecerse de las experiencias de los otros.

La modalidad de participación dentro de cada sesión también es de carácter voluntario, es decir, no se "obliga" a nadie a opinar o manifestarse sobre el tema abordado, validándose así que existen diferentes maneras de participar, ya sea a través de un juego o dinámica, escuchando, observando u opinando. Esto es muy reconocido y apreciado por los participantes, ya que se sienten "libres" dentro del taller, y al mismo tiempo esto les otorga mayor poder y responsabilidad acerca de los temas que se aborden, así como las conclusiones y consensos a los que se llegue.

\section{- Taller grupal "}

\section{Sesión 1}

\section{Objetivos Específicos:}

Que los(as) participantes logren:

1. Conocer los objetivos, metodología y estructura del taller

2. Internalizar el Concepto de "Hábito".

Esta primera sesión, en resumen, intenta favorecer un ambiente de confianza y motivación inicial en el proceso de taller, potenciando la expresión espontánea de sentimientos y emociones. Es importante aquí destacar la voluntariedad y el compromiso personal de la participación en la actividad grupal validando todas las formas de participar y no condicionando la asistencia al taller.

A través de técnicas de dinámica grupal y juegos, se facilita a los usuarios la posibilidad de expresar libre y espontáneamente su relación con los alimentos, intencionando un proceso de aceptación y autoconocimiento, al mismo tiempo, el T.O. va intencionando la reflexión y llevando al grupo a "definir" qué es un hábito, y además identificar algunos factores contextuales que los potencian y mantienen en el tiempo dentro de un grupo familiar. Con ello se persigue el explicitar o hacer evidentes las características socioculturales que subyacen a la ejecución de los hábitos alimenticios, a través de la práctica de las rutinas cotidianas, los ritos familiares y todas aquellas experiencias del quehacer validadas por nuestro entorno familiar, comunitario y social que no son "conscientes" y que de alguna manera están influyendo en nuestro estado de salud general[15]. Por otra, parte se busca también el favorecer o intencionar un proceso de toma de conciencia de los hábitos alimenticios presentes en los ritos familiares a través de la práctica. 


\section{Sesión 2}

Objetivos Específicos:

Que los(as) participantes logren:

1. Analizar y reflexionar acerca de los factores emocionales y afectivos relacionados con el hábito de alimentarse.

2. Reconocer el contexto familiar, social y cultural en el que se dan los hábitos.

3. Reconocer las características circulares y cíclicas de los comportamientos relacionados con la alimentación.

En la segunda sesión, y en continuidad con los elementos abordados en la sesión número uno, lo que se busca es favorecer proceso de análisis y reflexión acerca de los factores emocionales y afectivos del habito de alimentarse, visto en un contexto familiar y sociocultural. El T.O. invitará a los grupos a conversar y debatir acerca de la siguiente pregunta ¿Para qué comemos los seres humanos?. El objetivo es "valorizar" la importancia de aquellas respuestas relacionadas con lo afectivo, emocional, cultural y social de la alimentación, comparándolas con las respuestas relacionadas con lo biológico o físico a fin de rescatar la importancia significativa de las primeras en comparación con las segundas (generalmente las respuestas relacionadas con lo afectivo son muchas más en número, que las relacionadas con lo nutricional). También resultan ser elementos interesantes de la discusión el destacar cómo vemos estos aspectos en nuestra vida cotidiana y cuáles de las repuestas nos hacen más o menos sentido.

Para terminar la T.O. invitará a las participantes a reflexionar acerca de cómo se podrían enfrentar aquellas conductas que nos gustaría modificar o que nos provocan conflicto en relación al análisis anterior.

Lo anterior se desarrolla a partir de dos dinámicas grupales y un plenario.

\section{Sesión 3}

Objetivos Específicos:

Que los(as) participantes logren:

1. Analizar y reflexionar acerca del significado y función de los hábitos alimenticios en un contexto familiar.

2. Reconocer las influencias que han recibido de su familia de origen. (Proceso de Transmisión Multigeneracional).

A través del dibujo del genograma familiar, por parte de cada uno de los integrantes del grupo, se pretende facilitar un proceso de análisis en relación a la propia familia, destacando que el sentido que tiene el ejercicio es "poder mirar" a nuestra familia nuclear y de origen de manera gráfica, reconociendo así las influencias que han recibido de su familia de origen y a su vez cómo se están perpetuando y transmitiendo éstas en su familia actual. Además, en el dibujo se señalan con símbolos algunas patologías relacionadas con la temática del taller, tales como HTA, DM, obesidad, tabaquismo, ansiedad, etc., de manera que el instrumento sirva como un "mapa" 
histórico y transgeneracional que les permita a los participantes reconocer cómo estos factores de riesgo se han ido traspasando de generación en generación, y de esta manera generar estrategias para el cambio en este escenario. También se puede trabajar en relación a "diferenciar" aquellos factores donde predomina una herencia "biológica" o "de patrones de conducta heredados", donde el objetivo no es tender a "dicotomizar o polarizar" el análisis, sino más bien comprender cómo estos factores se relacionan y complementan para dar paso a nuestro "estilo de vida y factores de riesgo asociados".

\section{Sesión 4}

Objetivos Específicos:

Que los(as) participantes logren:

1. Visualizar y crear estrategias para el Autocuidado, desde su experiencia personal y lo aprendido en el taller.

En esta última sesión, lo que se busca es favorecer un proceso de "integración" de los conocimientos y experiencias adquiridas en el proceso de taller, las que serán concretizadas en un "producto". Se invita a los participantes a hacer un Afiche ilustrativo acerca de "Nuevas estrategias para desarrollar nuestro Autocuidado". Esto también se complementa con algunas actividades lúdicas y un plenario de cierre.

\section{- Conclusiones y resultados de la intervención "}

\section{Resultados Cuantitativos}

Del total de participantes (70), un 90,3\% corresponde a mujeres, y el 9,7\% corresponde a hombres.

Respecto a la baja en el peso corporal, se concluye que el $61,3 \%$ lograron bajar entre 1 y $5 \mathrm{Kg}$. El 22,5\% bajó entre 6 y 10 kilos, y el 4,8\% logró bajar entre 11 y 15 kilos. Eso resume que un total de 55 personas, bajaron de peso, lo que equivale al $88,6 \%$ de los participantes. Sólo 2 personas suben de peso correspondiendo al 3,22\% del total y 3 mantienen su peso inicial.

Respecto de la presión arterial, también se observaron cambios importantes respecto al ingreso y egreso del proyecto, observándose que aproximadamente un $25 \%$ de los usuarios, ingresaron con presiones superiores a 140/90, porcentaje que al momento de egreso no superaba al 6\%. Por último, también se midió la glicemia en ayunas, donde se observó que al ingreso, un $60 \%$ presentaba rangos mayores a $100 \mathrm{mg} / \mathrm{dl}$, porcentaje que al egreso no superaba el $19 \%$. 


\section{Resultados Cualitativos}

Para evaluar cualitativamente la experiencia, se fotografiaron algunas sesiones y se llevó una bitácora del proceso sesión a sesión. Además, en la finalización de cada taller, se realizó un plenario de evolución y una encuesta escrita, en la que se solicitaba a los usuarios que entregaran sus opiniones y sugerencias respecto a la intervención de Terapia Ocupacional.

Observamos que los usuarios participaron activamente en las sesiones grupales e individuales de Terapia Ocupacional, y creemos que esta adherencia se debe fundamentalmente a la motivación e interés que representaba este tipo de intervención y a que el horario establecido resultaba favorable con respecto a las actividades que desempeñan durante el día (trabajo, cuidado de los hijos, estudios, etc.). A su vez, las características de las sesiones de Terapia Ocupacional fomentaron la participación activa de los usuarios. Para ejemplificar de mejor manera este último aspecto podemos citar algunas frases textuales de los usuarios(as):

"A mi me pareció excelente las reuniones ya que pudimos compartir nuestras experiencias en grupo".

"En este taller he encontrado gente nueva y cariñosa que te escucha y te aconseja y te suben el ánimo, razonamos y discutimos los problemas que nos exponen".

A partir de estas reflexiones podemos darnos cuenta que el proceso de toma de conciencia fue satisfactorio, los usuarios lograron identificar aquellas actitudes y factores que influyen en su estado de salud - enfermedad actual.

"La terapia que realizamos la agradezco, uno aprende y hace conciencia de lo bueno y lo malo".

"El taller de Terapia Ocupacional es una gran ayuda para mi ya que los temas que tratamos nos permiten analizar el problema y crear soluciones, para entender lo que nos pasa".

"Me enseñó ha darme cuenta en lo que he estado fallando en forma simple y fácil y así poder enseñar a mi familia".

Los usuarios, a partir de su experiencia personal construyeron el concepto de hábito, como una tendencia latente que opera a nivel inconsciente y que tiene una significancia personal, es por ello que mientras no encuentren un sentido para el cambio de estos hábitos no saludables, no lograrán modificarlos. Por otro lado, descubrieron que esto es un proceso a largo plazo y que requiere esfuerzo, dedicación y perseverancia; tanto por parte de ellos como de la participación del entorno:

"Me ha servido para identificar factores que me producen buenos y malos hábitos, ..., si bien estos factores han existido siempre, con este taller los he podido identificar para realzar los que me benefician y eliminar los que me perjudican".

"Nos enseñan ha educar a nuestros hijos y formarles buenos hábitos y así criarlos en forma sana".. 
"Sugiero que no se acabe el taller de Terapia Ocupacional porque nos ayuda a salir de nuestros malos hábitos alimenticios".

A través del proceso llevado a cabo pudimos observar que los(as) participantes lograron identificar aquellas emociones que promueven un hábito alimenticio no saludable y que desean cambiar. Un ejemplo de esto es lo que se puede observar en los siguientes testimonios:

"Cada vez que estoy triste voy al refrigerador y me lo como todo".

"A veces no tengo hambre y como por ansiedad".

"Debo agradecer a la terapias de grupo que me ayudaron a encontrar las razones que nos llevan a comer y con esta a aprender a controlar mi ansiedad".

Los participantes identificaron las etapas correspondientes al ciclo de la ansiedad y a su vez en conjunto buscaron una solución con sentido para ellos y que les evite caer en ese círculo vicioso.

Un aspecto no considerado dentro de los objetivos de nuestra intervención, es el incremento de la autoestima por parte de los usuarios. El hecho de compartir experiencias con otras personas implica que no son los únicos en este proceso; por lo tanto sienten más apoyo y se valoran más como personas, encontramos aquí el fenómeno de lo que se denomina "universalización de los problemas", es decir, darse cuenta que no se es el único que tiene el problema, y por lo tanto de esta manera se favorece una mayor apertura frente al tema, mayor flexibilidad y creatividad para enfrentarlo y se eliminan algunos mecanismos de defensa que podrían operar en contra de un proceso de crecimiento y desarrollo.

Podemos observar entonces, que sin ser un objetivo especifico de la intervención, el aumento de la autoestima y la confianza en si mismo se da como un valor agregado y producto más bien de "la relación" que se establece entre Terapeuta y usuario, así como la relación que se da entre los mismos participantes, lo que genera un vínculo de confianza y respeto al interior del grupo.

"Es un programa súper bueno, ayuda a tener una autoestima mejor".

"Me sirvió mucho para valorarme a mi aún más como persona y a sentir más apoyo".

"Me sirvió a desarrollar mi personalidad y además saber mas de mis cosas y

entretenerme en grupo".

"Nos ayudó a conocer nuestros problemas de salud, aceptarnos y querernos como somos".

Finalmente, podemos mencionar que todos los aspectos concluidos anteriormente influyen en la baja de peso que los participantes en su mayoría experimentaron. Por esta razón sabemos que Terapia Ocupacional es parte fundamental del equipo multidisciplinario en el abordaje de la promoción de un estilo de vida saludable, a través del cambio de hábitos; el cual sólo se puede modificar desde una perspectiva basada en la Ocupación, ya que estos forman parte fundamental del Desempeño Ocupacional de cada persona.

Toda ocupación procede de una tendencia espontánea del ser humano de interactuar con su ambiente y modificarlo, e implica el desenvolvimiento y desempeño en las distintas tareas que dan significado a una necesidad individual de sentirse competente y satisfecho al pertenecer a un mundo social y a validar sus propias capacidades, 
intereses y valores personales adquiridos en experiencia, además de influir directamente en la salud biológica, mental y psicosocial del individuo.

Por lo tanto, al ser la Terapia Ocupacional la única disciplina que centra el abordaje de los problemas del individuo desde una mirada biopsicosocial sistémica, y que toma en cuenta todos los aspectos involucrados en el Desempeño Ocupacional del ser humano, es decir, lo histórico, social, cultural, el ambiente físico y las habilidades y características particulares y únicas de cada individuo, es que ella no puede estar ausente en el abordaje integral del proceso de modificación de hábitos y otros aspectos que impliquen un cambio significativo para la persona, sobre todo si nuestra expectativa como equipo de salud, es que ese cambio sea sustentable en el tiempo y que favorezca en el individuo el lograr sentirse eficaz consigo mismo y su entorno familiar, social y comunitario.

Como factores limitantes de la experiencia, podemos mencionar el número de sesiones, ya que, en primera instancia el taller grupal se planificó con un número de 8 , sin embargo, por un tema de presupuesto, FONASA aprobó sólo 4 sesiones por grupo (situación que también ocurrió con el numero de sesiones propuestas por otros profesionales del equipo).

Creemos que con un número mayor de sesiones y manteniendo las instancias de coordinación y seguimiento por parte del equipo de salud a cargo del programa, se potenciarían los resultados de la experiencia.

Cabe señalar, que al ser los participantes de proyecto, también usuarios del CESFAM, esto ha facilitado el seguimiento de cada uno de ellos y el abordaje oportuno de "recaídas" o dificultades que pudiesen presentarse posterior al egreso del programa. Sin embargo, estimamos que para favorecer la integralidad del proceso, el proyecto debiese contemplar dentro de sus actividades, el seguimiento a largo plazo.

\section{Agradecimientos "}

A Claudia Martínez, Nutricionista, Jorge Padilla, Kinesiólogo, Nélida Maldonado, Técnico Administrativo, Elisa Molina Auxiliar Paramédico y a todos los participantes del proyecto que compartieron sus testimonios y experiencias para publicar este articulo. 


\section{Referencias}

(1) MINSAL. Los Objetivos Sanitarios para la Década 2000- 2010. División de Rectoría y Regulación Sanitaria. Departamento de Epidemiología, Primera Edición, Octubre de 2002.

(2) BERRÍOS X. Proyecto Interhealt - OMS- CHILE. Estudio de Prevalencia de factores de riesgo de enfermedades crónicas en población adulta mayor de 15años. Región Metropolitana, Chile, 1998, 1992, 1997. Datos no publicados.

(3) BOWEN, M. De la Familia al Individuo. Editorial Paidos, Barcelona. 1991.

(4) BRONFENBRENNER, U. La Ecología del Desarrollo Humano, Editorial Paidós. Barcelona, 1987.

(5) KIELHOFNER, G. Model of Human Occupation, Theory and Application. $3^{\text {rd }}$. Ed. United States of America. 2002.

(6) FREIRE, P. Pedagogía del Oprimido. Siglo XXI Editores. México 1999.

(7) WATZLAWICK, P.; HELMICK, B. y JACKSON, D. Teoría de la comunicación humana; interacciones, patologías y paradojas, Editorial Herder, Barcelona, 1983.

(8) GUBBINS, BERGER, Pensar en Desarrollo Familiar. Una Perspectiva Transdisciplinaria. Editado por Universidad Alberto Hurtado. Santiago 2004.

(9) MORÍN. E.. El (a)Método, 1921. Apuntes: Diplomado estrategias para el Desarrollo de las Familias U. Alberto Hurtado. Escuela de Psicología EneroDiciembre 2003.

(10) PASMIÑO H., PORRAS X., ÚBEDA C., Paciente Difícil. Recursos del Profesional en Jaque. Revista Chilena Medicina Familiar. 2004; Vol 5, № 3: 103 - 108.

(11) WATZLAWICK, P.; WEAKLAND, J., y FISCH, R. Cambio; Formación y Solución de los Problemas Humanos. Editorial Herder, Barcelona, 1987.

(12) AOTA. Occupational Therapy Practice Framework: Domain and Process, Draft XVIII. 2002. 
(13) AYALES I. et al. Haciendo Camino al Andar: Guía Metodológica para la Acción Comunitaria. OEF Internacional Washington DC, 1991.

(14) Mc GOLDRIC y GERSON. Genogramas en la Evaluación Familiar. Editorial Gedisa 1985.

(15) LAPLANTINE F. Antropología de La Enfermedad. Ediciones del Sol. Buenos Aires 1981. 